\title{
Terra MODIS Band 27 Electronic Crosstalk Effect and Its Removal
}

\author{
Junqiang Sun ${ }^{\mathrm{a}}$, Xiaoxiong Xiong ${ }^{\mathrm{b}}$, Sriharsha Madhavan $^{\mathrm{c}}$, and Brian Wenny ${ }^{\mathrm{a}}$ \\ ${ }^{\text {a }}$ Sigma Space Corp., 4600 Forbes Blvd., Lanham, MD 20706 \\ ${ }^{\mathrm{b}}$ Sciences and Exploration Directorate, NASA/GSFC, Greenbelt, MD 20771 \\ 'Science and Systems Applications Inc, 10210, Greenbelt Road, Lanham, MD 20706
}

\begin{abstract}
The MODerate-resolution Imaging Spectroradiometer (MODIS) is one of the primary instruments in the NASA Earth Observing System (EOS). The first MODIS instrument was launched in December, 1999 on-board the Terra spacecraft. MODIS has 36 bands, covering a wavelength range from $0.4 \mu \mathrm{m}$ to $14.4 \mu \mathrm{m}$. MODIS band 27 (6.72 $\mu \mathrm{m})$ is a water vapor band, which is designed to be insensitive to Earth surface features. In recent Earth View (EV) images of Terra band 27, surface feature contamination is clearly seen and striping has become very pronounced. In this paper, it is shown that band 27 is impacted by electronic crosstalk from bands 28-30. An algorithm using a linear approximation is developed to correct the crosstalk effect. The crosstalk coefficients are derived from Terra MODIS lunar observations. They show that the crosstalk is strongly detector dependent and the crosstalk pattern has changed dramatically since launch. The crosstalk contributions are positive to the instrument response of band 27 early in the mission but became negative and much larger in magnitude at later stages of the mission for most detectors of the band. The algorithm is applied to both Black Body (BB) calibration and MODIS L1B products. With the crosstalk effect removed, the calibration coefficients of Terra MODIS band 27 derived from the BB show that the detector differences become smaller. With the algorithm applied to MODIS L1B products, the Earth surface features are significantly removed and the striping is substantially reduced in the images of the band. The approach developed in this report for removal of the electronic crosstalk effect can be applied to other MODIS bands if similar crosstalk behaviors occur.
\end{abstract}

Keywords: MODIS, Moon, Crosstalk, Terra, Thermal emissive bands, Striping

\section{INTRODUCTION}

The Moderate Resolution Imaging Spectroradiometer (MODIS) is a key instrument for NASA's Earth Observing System (EOS). ${ }^{1-3}$ Two MODIS instruments are currently on-orbit, one onboard the Terra spacecraft launched on December 18, 1999 and the other onboard the Aqua spacecraft launched on May 4, 2002. The MODIS is a crosstrack scanning radiometer with 36 spectral bands covering a spectral range from 0.4 to $14.4 \mu \mathrm{m}$. There are three spatial resolutions (nadir) and numbers of detectors per band: $250 \mathrm{~m}$ (bands 1-2) with 40 detectors, 500m (bands 3-7) with 20 detectors, and $1 \mathrm{~km}$ (bands 8-36) with 10 detectors. The 36 spectral bands are distributed on four focal plane assemblies (FPA): visible (VIS), near-infrared (NIR), short- and mid-wave infrared (SMIR), and long-wave infrared (LWIR). Among the 36 bands, 22 are reflective solar bands (RSB) and 16 are thermal emissive bands (TEB). The RSB are calibrated by an on-board solar diffuser (SD) with its degradation monitored using an on-board SD stability monitor (SDSM) and by monthly lunar observations. ${ }^{4,5}$ The TEB are calibrated by an on-board black body (BB) ${ }^{6,7}$

MODIS band $27(6.72 \mu \mathrm{m})$ is a water vapor band which is insensitive to Earth surface features. ${ }^{8}$ Early in the mission, surface features with large temperature contrasts, such as islands or peninsulas were hardly seen in the Earth view (EV) images of Terra band 27. In 2004, band 27 began to show signs of surface feature contamination which has become more evident in subsequent years. After 11 years of nearly continuous operations, the band 27 EV images clearly show surface features which has noticeable impacts on MODIS higher level products. This paper uses MODIS EV images over the Baja Peninsula region for analysis. With the Earth surface feature contamination 
seen more and more clearly in EV images of Terra band 27, the striping due to detector differences has become stronger over the lifetime of the sensor. Early in the mission, the top-of-atmosphere (TOA) temperature detector differences among the ten detectors of Terra band 27 is less than $1.5 \mathrm{~K}$. The differences increase with lifetime and are currently as large as $10 \mathrm{~K}$ in recent images over land. In this paper, it is shown that both the occurrence of the EV surface features and the striping in Terra band 27 EV images are due to the electronic crosstalk from bands 28$30(7.33$ to $8.55 \mu \mathrm{m})$, among which band 29 is a surface-sensititive band. It is also shown that the crosstalk pattern and strength have significantly changed since launch, especially in last few years, which results in clear surface features and strong striping in Terra band $27 \mathrm{EV}$ images.

Electronic crosstalk was a critical issue for Terra MODIS SWIR/MWIR bands (1.24 to $4.52 \mu \mathrm{m}$ ) and was investigated during pre-launch testing and evaluated on-orbit early in the mission. ${ }^{9}$ Several algorithms were developed to remove the crosstalk contamination in these bands. Due to the large magnitude of the crosstalk and the many bands involved, these algorithms provided limited success. Terra band $2(0.85 \mu \mathrm{m})$ was also found to have electronic crosstalk from band $16(0.87 \mu \mathrm{m})$, resulting in two sets of very regularly distributed dots in the Earth scene images of band 2. Since the crosstalk in band 2 involves only band 16, it is much easier to be addressed compared to the crosstalk in the SWIR/MWIR bands. A linear algorithm was developed to describe the crosstalk in band 2. ${ }^{10}$ By applying the algorithm with the crosstalk coefficients derived from the lunar observations, ${ }^{11}$ the dotted features in EV images of Terra band 2 are significantly reduced as long as the crosstalk sender, band 16, is not saturated. ${ }^{10}$ Since MODIS band 16 is designed for ocean applications, it saturates frequently when it views the land. Considering MODIS band 2 is a land band, further investigation for the cases when band 16 saturates is required before the algorithm can be applied to MODIS L1B data.

MODIS band 27 is a thermal emissive band and located on the LWIR FPA, ${ }^{6,7}$ as seen in Figure 1. Bands 28, 29, and 30 are also located on the LWIR FPA. For Terra MODIS, there are crosstalk effects among these four bands. In this paper, we focus on the characterization and mitigation of crosstalk for Terra band 27. Fewer bands are involved compared to the crosstalk in the SWIR/MWIR bands and the involved bands are not saturated in both calibrator (BB) and EV observations compared to the crosstalk in the band $2 .^{10}$ In section 2, a linear algorithm is described for the crosstalk among MODIS bands. In section 3, the crosstalk coefficients for the crosstalk of Terra band 27 from bands 28-30 are derived from Terra MODIS lunar observations. They show that the crosstalk is strongly detector dependent and changes with the lifetime of the sensor. In section 4, the crosstalk correction is applied to remove the crosstalk contamination in the calibration coefficients derived from the BB observations and the calibration coefficients show less variability and detector differences become smaller after the correction. In section 5, the crosstalk correction is applied to EV data to reduce the crosstalk contributions from bands 28-30. The Earth surface features, such as the Baja peninsula, are significantly removed and the striping is substantially reduced in the EV images of the band after the correction is applied. The algorithm developed in this report for removal of the electronic crosstalk effect can be applied to other MODIS bands as well as to other instruments as long as the involved bands are not saturated.

\section{CROSSTALK CORRECTION ALGORITHMS}

Electronic crosstalk is a phenomenon of electromagnetic induction among the signals produced by different bands on the same FPA. It is a very complex issue and difficult to describe. As mentioned in the last section, in order to extract the cross-talk signal reliably, one of the requisites is that the lunar response does not saturate. This assumption is violated in most thermal emissive bands with the exceptions of band 21 and 31 respectively. Therefore, the lunar responses are ratioed to the non-saturating band 31 to mimic the actual lunar response for the other bands. As a first-order approximation, the crosstalk contribution from a sending band $\mathrm{B}_{\mathrm{s}}$, detector $\mathrm{D}_{\mathrm{s}}$ to a receiving band $B_{r}$, detector $D_{r}$ can always be expressed to be a linear function of the background subtracted instrument response of band $\mathrm{B}_{\mathrm{s}}$, detector $\mathrm{D}_{\mathrm{s}} .{ }^{10}$ For the rest of the discussions in this paper the detector $\mathrm{D}$ is referred in $\mathrm{L} 1 \mathrm{~B}$ product order. The total crosstalk contributions to band $\mathrm{B}_{\mathrm{r}}$, detector $\mathrm{D}_{\mathrm{r}}$ can be written as

$$
d n_{B_{r} D_{r}}^{\text {xtalk }}(F)=\sum_{B_{s}, D_{s}} c\left(B_{r}, D_{r}, B_{s}, D_{s}\right) d n_{B_{s} D_{s}}\left(F+\Delta F_{r s}\right)
$$

where $F$ is the frame of $B_{r}, \Delta F_{r s}$ is the distance between receiving band $B_{r}$ and sending band $B_{s}$ on the focal plane where they are located, $c\left(B_{r}, D_{r}, B_{s}, D_{s}\right)$ is the crosstalk coefficient for the crosstalk from band $B_{s}$, detector $D_{s}$ to band 
$\mathrm{B}_{\mathrm{r}}$, detector $\mathrm{D}_{\mathrm{r}}$, and $d n_{B_{s} D_{s}}\left(F+\Delta F_{r s}\right)$ is the background subtracted measured instrument response of band $\mathrm{B}_{\mathrm{s}}$, detector $\mathrm{D}_{\mathrm{s}}$ at frame $\mathrm{F}+\Delta \mathrm{F}_{\mathrm{rs}}$. The electronic crosstalk occurs only among the bands on the same FPA. Thus, the summation for the receiving band in Eq. (1) is only over the bands on the FPA where the receiving band is located.

MODIS thermal emissive bands are all $1 \mathrm{~km}$ bands and each has 10 detectors. Since the response of the Earth view in this thermal wavelength is uniformly distributed for each detector within a scan, Eq. (1) can be further approximately expressed as

$$
d n_{B_{r} D_{r}}^{\text {xtalk }}(F)=\sum_{B_{s}} C\left(B_{r}, D_{r}, B_{s}\right)\left\langle d n_{B_{s} D_{s}}\left(F+\Delta F_{r s}\right)\right\rangle_{D_{s}},
$$

where the brackets indicate the average over the ten detectors of the sending band $B_{s}$ and $C\left(B_{r}, D_{r}, B_{s}\right)$ is the effective crosstalk coefficient from the sending band $B_{s}$ to band $B_{r}$, detector $D_{r}$. Since the gains of the detectors are not exactly the same, responses of the ten detectors are different even if they view a homogeneous surface. Thus, $C\left(B_{r}, D_{r}, B_{s}\right)$ is not a simple summation of $c\left(B_{r}, D_{r}, B_{s}, D_{s}\right)$ over $D_{s}$. It is a weighted summation of the sending detector dependent crosstalk coefficients. As mentioned above, the scene brightness temperature usually does not change dramatically within a scan, hence, Eq.(2) can be effectively applied in MODIS L1B for EV scenes.

The instrument response with removal of the crosstalk contamination can be written as

$$
d n_{B_{r} D_{r}}^{\text {corr }}(F)=d n_{B_{r} D_{r}}(F)-d n_{B_{r} D_{r}}^{\text {xtalk }}(F)
$$

where $d n_{B_{r} D_{r}}(F)$ is the measured background subtracted instrument response for band $B_{\mathrm{r}}$, detector $D_{\mathrm{r}}$. The correction described in Eqs. (1) - (3) should be applied to both L1B production and black body (BB) calibration. To apply the above equations to remove the impact of the electronic crosstalk, one has to obtain the crosstalk coefficients. The crosstalk coefficients can be measured prelaunch although other effects such as scattering light may strongly affect the accuracy of the measurements. However, the crosstalk effect may change on-orbit and the crosstalk coefficients need to be updated frequently.

\section{CROSSTALK COEFFICIENTS}

The Moon is a point-like light source and is very suitable for the analysis of remote sensor on-orbit crosstalk effects. ${ }^{11}$ Figure 2 shows the lunar image observed by Terra MODIS band 27 detector 1 on July 22, 2008. The image is the background subtracted instrument response of the detector. To see the crosstalk effect more clearly, the z-axis maximum is restricted to $200 \mathrm{DN}$. Thus, the center of the image is not the real image of the Moon. In fact, we are only interested in the edges of the lunar image since impact due to the crosstalk can be seen. The size of the Moon image is nearly 7 pixels of a MODIS $1 \mathrm{~km}$ band. The deep valley on the left side is the effect of the crosstalk. Since its contribution is negative, it can only be electronic crosstalk. According to positions of the bands on the LWIR FPA, the crosstalk effect seen in Figure 2 can only come from bands 28-30. Crosstalk can only occur among bands on the same FPA. Thus, band 27 can have crosstalk, in principle, from bands 31-36 since they all are on the LWIR FPA. If there is crosstalk from bands 31-36 for band 27, the crosstalk contributions should be seen at the right edge of the lunar image in Figure 2 according their positions on the LWIR FPA shown in Figure 1. From the lunar image in Figure 2, no obvious crosstalk contributions are seen from bands 31-36.

The Moon is not a homogenous surface. In one scan the detectors of a given MODIS band view different portions of the lunar surface and will show a radiance difference among detectors. However, a single detector lunar image can be constructed from multiple scans thus each detector will view every region of the lunar surface. A sum can be calculated for the background subtracted instrument response for each detector over scans and the values of the summation only depend on frame. Figure 3 shows the summations of each Terra MODIS band 27 detector responses for the lunar view on July 22, 2010 (y-scale cut-off at 4000 DN). Frames 19-24 are the peaks of the lunar response and the structure seen to the left of frame 19 is due to the crosstalk effects. The plots in the figure demonstrate that the crosstalk effects are different for the individual detectors. For most of the detectors, the contributions of the crosstalk are negative while for a few detectors, they are positive. 
The Moon is a point-like light source, not a true point source. Its size is about 7 MODIS 1 km pixels in diameter as viewed from orbit. Since the distance between two adjacent detectors of a MODIS $1 \mathrm{~km}$ band is only one pixel, several detectors in each MODIS band view the Moon at the same time during a lunar observation although they view different parts of the Moon. It is very difficult to separate the crosstalk contributions from different detectors of the same band. As mentioned in section 2, the retrieved brightness temperature of the EV surface is very uniform for the detectors within each scan, and for the cross-track response of a MODIS scan at nadir. With this approximation, only the total crosstalk contribution of a sending band for a smooth scene is of interest. The Moon provides a light source equivalent to a smooth surface if their background subtracted responses are summed over scans for a complete lunar observation. The summations displayed in Figure 3 can be used to derive the crosstalk coefficients which are only dependent on the sending band for each detector of band 27.

The distance between the two adjacent bands on the LWIR FPA is either 3 pixels or 4 pixels, which is still smaller than the size of the Moon, which is about 7 pixels in diameter. This means there are superimpositions among the crosstalk contributions of sending bands which are adjacent on the LWIR FPA. To separate the crosstalk contributions from different sending bands, the crosstalk coefficients are derived for the band with the largest distance from band 27 first. From Figure 1, bands 28-30 are on left side of band 27 and other bands on the LWIR FPA are on the right side of band 27. Band 30 is 9 pixels away from band 27, the furthest of band of all to the left of band 27. Thus, the crosstalk coefficients from band 30 to band 27 are derived first. For bands on the right side of band 27, Figure 3 shows that their crosstalk contributions to band 27 are negligible compared to those from bands 28-30. In this paper, the focus is on the crosstalk contributions from bands 28-30 to band 27.

As seen in Figure 3 there are about 7 frames of band 27 that have crosstalk contributions from band 30 considering the size of the Moon is about 7 pixels in diameter. Among these 7 frames, half have contributions from band 30 only and other half have contributions from both band 30 and band 29. The frames which have contributions only from band 30 are used to derive the crosstalk coefficients for the crosstalk from band 30. The derived coefficients are then applied to remove the crosstalk contributions from band 30 in the summations of the instrument responses of band 27. The crosstalk effect from band 30 is removed not only in the frames used to derive the coefficients but also in the frames where band 29 has crosstalk contributions. In the corrected summations of the instrument responses, there are no electronic crosstalk contributions from band 30 in principle. Then we can find 7 frames of the corrected data which have crosstalk contributions from band 29. Similar to band 30 in the original summations of the instrument responses, half of them are purely crosstalk contributions from band 29 and the other half are the combinations of the crosstalk contributions from both band 29 and 28 . The former can be used to derive the crosstalk coefficients for band 29. With derived crosstalk coefficients for band 29, we can repeat used to bands 30 and band 29 to derive the crosstalk coefficients for band 28.

Figures 4-6 show the derived crosstalk coefficients for the crosstalk of Terra band 27 from bands 28, 29, and 30, respectively. They are derived from the monthly lunar observations over the instrument lifetime. From the plots, it is seen that Terra band 27 has non-negligible crosstalk from all three bands. Although the coefficients have some short-term fluctuations, they are stable over the long-term, indicating that the crosstalk impacts may be correctable. The crosstalk coefficients are strongly detector dependent. Their detector dependence means that the crosstalk impacts individual detectors differently, which results in striping in EV images. For most detectors, the crosstalk effects change with time smoothly. However, the crosstalk effects have sudden changes for a few detectors, especially detector one. For detector one, the crosstalk contributions from all three bands changed from positive to negative around year 2004. Due to this crosstalk pattern change, the striping in band $27 \mathrm{EV}$ images should become much stronger after 2004.

The crosstalk coefficients are derived using the same procedure applied to bands 28-30 for bands 31-36 which are also located on the LWIR FPA. The derived coefficients are around 0.002, which result in a much smaller impact on band 27 compared to those from bands 28-30. This is expected since the right side of the lunar image in Figure 2 is smooth, which indicates that bands 31-36 does not significantly contribute to the cross talk as mentioned earlier. The reason for the negligible cross talk from bands 31-36 is due to the fact these channels are photo-conductive in nature while bands 27-30 are photo-voltaic. When the crosstalk coefficients shown in figures 4-6 are applied to BB calibration and the L1B EV products, six-month averaged values are used to reduce the short-term fluctuations. At 
the range where there is a sudden jump for a coefficient, only the data at same side of the jump are used for the average.

Figure 7 shows the same lunar observation as shown in Figure 3 except with the crosstalk correction applied. The improvement is evident by the absence of the structure on the left side of the lunar image that was seen in Figure 3. This demonstrates that the algorithm described in section 2 is effective. The curvature of the tail at the right side of the lunar image in figure 7 is not due to the crosstalk effect but the brightness variation caused by the phase variation of the moon.

\section{CROSSTALK CORECTION IN BLACK BODY CALIBRATION}

MODIS thermal emissive bands are calibrated on-orbit using an on-board BB. A quadratic approximation is applied to describe the relationship between the incident radiance and the instrument response for each MODIS thermal emissive band, ${ }^{6,7}$ that is,

$$
L_{B D}=a_{0}(B, D)+b_{1}(B, D) d n_{B D}+a_{2}(B, D) d n_{B D}^{2}
$$

where $L_{B D}$ is the radiance received by band $B$ detector $D, a_{0}(B, D)$ and $a_{2}(B, D)$ are offset and nonlinear term coefficient of band $\mathrm{B}$ detector $\mathrm{D}$ which were derived prelaunch and updated on-orbit using the quarterly BB warmup and cold-down (WUCD) measurements, and $b_{1}(B, D)$ is the linear calibration coefficient of the detector of the band which is determined on a scan-by-scan basis in the MODIS L1B products. Since the offset and nonlinear term contributions in Eq. (4) are small quantities compared to that of the linear term, we focus on the impact of the crosstalk on the linear calibration coefficient.

The calibration coefficients of Terra band 27 without correction for the crosstalk effect are shown in Figure 8. They are calculated using the BB data on a scan-by-scan basis with averaging over the 203 scans in one granule. The offset, $\mathrm{a}_{0}(\mathrm{~B}, \mathrm{D})$, and nonlinear coefficient, $\mathrm{a}_{2}(\mathrm{~B}, \mathrm{D})$, used in the calculation are exactly the same as those used in the current Collection 6 L1B products. The coefficients trends are calculated using one granule for each 100 days for all ten detectors of the band since launch. It is seen clearly in the plot that the coefficients of detectors 1, 2, 3, 5, 7, and 8 have sudden jumps which induce discontinuities for the coefficients. The discontinuity occurs at different times for different detectors. The calibration coefficients after the correction of the crosstalk are shown in figure 9. The discontinuities are still present but are smaller in magnitude. The detector differences of the calibration coefficients in figure 9 are also smaller compared to those in figure 8. Figures 8 and 9 demonstrate that crosstalk has a significant impact on the calibration coefficients of Terra band 27, which can be as large as a few percent, results in discontinuities in the calibration coefficients, and increases the detector differences of the coefficients. They also demonstrate that the crosstalk impact in Terra band 27 linear calibration coefficients can be significantly reduced using the crosstalk coefficients derived from the lunar observations with the algorithms described in Section 2.

The calibration coefficients shown in figures 8 and 9 are the values averaged over scans in one granule. In MODIS L1B products, calibration coefficients are calculated on a scan-by-scan basis but averaged over 40 scans, 20 before and 20 after current scan. The forty-scan averaged calibration coefficients calculated without and with the crosstalk removed in one orbit on June 23, 2010 are displayed in Figures 10 and 11, respectively. Once again, they demonstrate that the crosstalk has a significant impact on the calibration coefficients and the algorithms described in section 2 and the coefficients derived from the lunar observations can substantially reduced the crosstalk impact on the coefficients.

\section{CROSSTALK CORECTION IN L1B EV PRODUCTS}

MODIS band 27 is primarily an atmosphere-sensing band and should not be very sensitive to Earth surface features. Terra MODIS has been on-orbit for more than ten years since launch on December 18, 1999. ${ }^{3}$ Early in the mission, Earth surface features were not evident in Terra band 27 images. After several years of operation, Earth surface features started to show up in scenes with high temperature contrast such as the Baja peninsula. This behavior has grown over time and the Baja peninsula can be clearly identified in recent Terra MODIS band 27 Earth View (EV) 
images. This surface feature contamination of band 27 images has begun to have an impact on higher level products, such as the MODIS water vapor products. Early in the mission, striping in Terra band 27 images is not particularly evident. Over the mission lifetime, the striping in the band's images has increased and become very strong in the last few years, predominantly caused by detector differences. As shown in sections 3 and 4, Terra band 27 has crosstalk from bands 28-30, the crosstalk pattern changes with time, and crosstalk became much stronger recently. In section 4, the crosstalk removal algorithms described in section 2 were applied to remove the crosstalk impact on calibration coefficients, resulting in remarkable improvement of the quality of the coefficients. In this section, the crosstalk removal algorithms and the crosstalk coefficients derived in section 3 are applied to Terra MODIS band 27 L1B products and show that both the Earth surface features and striping can be substantially reduced.

Terra MODIS band 27 collection 5 Earth surface brightness temperature observed on June 23, 2010 over the Baja peninsula area is shown in Figure 12. The same image with the crosstalk effect corrected is displayed in Figure 13. The image in figure 12 is a standard MODIS L1B product and with no crosstalk removal algorithms applied to remove the crosstalk effect. In this image, the Baja peninsula occurs as a dark shadow, where the measured brightness temperature is lower than other surrounding area. The dark shade is mainly induced by the crosstalk from band 29 which is a surface viewing band. Since the crosstalk contributions from band 29 to band 27 are negative, the crosstalk effect induces the dark shadow of the Baja peninsula area. Strong striping along the track direction is also seen in the image. As shown in figures 4-6, the crosstalk impact is strongly detector dependent which results in the striping along the track direction. With the crosstalk effect corrected, the feature of Baja peninsula and strong striping seen in Figure 12 has been greatly reduced. The crosstalk correction does not completely remove the surface feature.

Figure 14 shows the brightness temperature variation along the scan direction passing through the Baja peninsula observed by detector 1 with the along track index in the image being 720 in Figures 12 and 13 . The dotted curve is the brightness temperature without the crosstalk effect corrected and solid curve is the brightness temperature with crosstalk effect corrected. In the dotted curve, there is a sudden decrease in the brightness temperature around frame 880 which corresponds to the coastal edge of the Baja Peninsula. The brightness temperature continues at the decreased level over the width of the peninsula. Since band 29 is a land viewing band (or any appropriate name), its instrument response changes dramatically crossing the edge and high response over the peninsula. Thus, the crosstalk contributions from band 29 to band 27 are larger in magnitude over the range from frame 880 to 1000 . Since the crosstalk contributions from band 29 to band 27 detector 1 is negative, they induce a sudden decrease around frame 880 and a resulting lower temperature in the range from frame 880 to 1000 in the measured brightness temperature. Compared to the dotted curve, the solid curve in the plot is flatter as expected over the region from frame 880 to 1000 where the detector scans over the peninsula.

Figure 15 shows the brightness temperature variation along the track direction at the center frame in Figures 12 and 13, the dotted curve for the temperature without crosstalk correction and solid curve with the crosstalk effect corrected. In the dotted curve, the striping due to detector difference can be as large as $10 \mathrm{~K}$ and striping varies with location. This means that the striping cannot be removed by simply adjusting the calibration coefficients. The striping is not induced by the errors in the calibration coefficients derived from the BB observations. Over the Baja Peninsula, the left side of Figure 15, band 29 has a larger response and thus a larger crosstalk contribution to band 27 , which induces stronger striping. Once over the ocean, the instrument response of band 29 is relatively small and then less crosstalk contributions from band 29 to band 27, which result in smaller striping in the area. With the crosstalk effect removed, the striping is greatly reduced as shown in solid curve in Figure 15, where striping in brightness temperature is less than $2 \mathrm{~K}, 5$ times smaller than that observed in the top chart.

Crosstalk is a very complex phenomenon. The crosstalk removal algorithms have to be tested on many cases, different times and different scenes. Figures 16 and 17 show Terra MODIS band 27 collection 5 Earth surface brightness temperature observed on November 19, 2006 over the Baja peninsula area and the same image with the crosstalk effect corrected, respectively. In figure 16, the Baja peninsula is observable although not as evident as in Figure 12 and striping is remarkable although not as strong. Similar to figure 13, the application of the crosstalk correction improves the performance of the band 27, as seen in figure 17. Figure 18 show the brightness temperature variation along scan direction passing over Baja peninsula observed by detector 1 with the 
corresponding along track index being 2230 in Figures 17 and 18, the dotted curve without the crosstalk effect corrected and the solid curve with crosstalk effect corrected. The curve with the crosstalk effect corrected has a much flatter shape over the peninsula compared to that without the correction. Figure 19 shows the brightness temperature variation along the track direction, dotted curve without crosstalk correction and solid curve with the crosstalk effect corrected. In the dotted curve, the striping due to detector differences can be as large as $6 \mathrm{~K}$. In the solid curve, the striping is reduced to be less than $1.5 \mathrm{~K}$ after the crosstalk effect is removed. Thus, the crosstalk algorithms developed in Section 2 and the crosstalk coefficients derived in Section 3 work well not only in 2010 but also in 2006 although the crosstalk patterns are quite different in 2006 and 2010 as seen in Figures 4-6.

The Baja peninsula was not observed by Terra band 27 and the striping was not as noticeable early in the mission. However, the crosstalk coefficients shown in Figures 4-6 indicate that the crosstalk contributions from bands 28-30 to band 27 are not negligible. Compared to crosstalk contributions later in the mission, they are positive instead of negative for all detectors except detector 6 . In fact, the crosstalk coefficients for detector 6 are much smaller in magnitude compared to those of other detectors. Another feature of the crosstalk coefficients early in the mission is that the detector differences are much smaller than those later in the mission, which could explain why the striping was not very noticeable. Figures 20 and 21 show Terra MODIS band 27 collection 5 Earth surface brightness temperature observed on December 20, 2000 over the Baja peninsula area and the same image with the crosstalk effect corrected, respectively. In both images, the Baja peninsula is hardly seen or seen with a clear edge as seen in figure 12 and the striping is much smaller compared to that in Figures 12 and 15 . Figure 22 shows the brightness temperature variation along scan direction passing over the Baja peninsula observed by detector 1 with along track index being 1750 in Figures 20 and 21, the dotted curve without the crosstalk effect corrected and the solid curve with crosstalk effect corrected. The shapes of the two curves in Figure 22 look about the same. However, careful inspection shows that one can find the temperature around frame 800 is reduced more than other areas by the crosstalk correction. The Baja peninsula is more visible in Figure 21 than in Figure 20 although the difference is small. This means the brightness temperature of band 27 over Baja peninsula is slightly lower than over oceans. This is understandable since band 27 is a water vapor band. In fact, Figures 13 and 16, the peninsula is still visible after the crosstalk correction due to lower brightness temperature compared to the surrounding area. The crosstalk contributions from bands 28-30 to band 27 are positive early in the mission and negative later in the mission, which made Baja peninsula more invisible to band 27 early in the mission and more visible later in the mission. Figure 23 shows the brightness temperature along the track direction. As expected, striping is not significant whether the crosstalk effect is corrected. However, crosstalk correction still reduces the striping.

In the temporal analysis of the scene corrections it is observed that the removal of cross talk would affect the radiometry of the retrieved quantities. In the instance of corrections early in life the cross talk correction would decrease the brightness temperature by about $2 \mathrm{~K}$ on average and as the scene correction is performed for later years the brightness temperature would increase by about $2 \mathrm{~K}$ on average. This is expected as the radiometry is being corrected through the actual magnitude of the cross talk coefficients. In the early years, the coefficients are for almost all detectors positive number whereas most of them change direction into negative quantities and hence the reversal. The crosstalk correction is applied to both BB and EV. Whether the correction for EV brightness temperature is positive or negative not only depends on the signs of the coefficients but also the temperature difference of the BB and the EV site. Temperature of Baja peninsula in the cases analyzed in this paper is around $250 \mathrm{~K}$, while that of Terra BB is fixed at $290 \mathrm{~K}$. This means the temperature of the Baja peninsula is lower in cases than that of $\mathrm{BB}$ in the cases we analyzed here. For the area which has a temperature higher than that of $\mathrm{BB}$, the sign of the correction could be opposite. This means the average of the correction of the crosstalk effect to the brightness temperature of Terra band 27 over the entire dynamic range of the band and all sites may not necessarily be positive or negative. We will address this issue in detail elsewhere.

The crosstalk pattern has changed a lot during the mission lifetime as shown in Figures 4-6 and the changes are strongly detector dependent. The three examples shown above demonstrate that the crosstalk removal algorithm described in Section 2 and the crosstalk coefficients derived from the lunar observations work consistently well in early, middle, and late mission. We have also applied the crosstalk removal approach to other random scenes. It works on them as well as on the examples shown above. Thus, the crosstalk effect can be well described with linear approximation as described in Section 2 and the crosstalk coefficients derived from the lunar observations are quite 
accurate to account for the crosstalk effect. The algorithms developed in this report and the coefficients derived improve the quality of the Terra band 27 L1B products remarkably.

\section{SUMMARY}

Algorithms are developed to describe electronic crosstalk among MODIS bands on the same focal plane. In the algorithms, a linear approximation is used to relate the crosstalk contribution and the sending band's instrument response. The algorithms can be applied to remove the impact of the crosstalk effect to any band which has crosstalk from other bands. The algorithms should be applied to both calibrators and the MODIS L1B products. The crosstalk coefficients with the linear approximation can be derived from lunar observations. The approach has been applied to Terra MODIS band 27, which has crosstalk from bands 28-30. The crosstalk coefficients for the crosstalk from the three bands to band 27 are derived from the scheduled Terra lunar observations. The crosstalk contributions from these bands to band 27 are strongly detector dependent and change with time, dramatically sometimes. The crosstalk contributions had positive values to the measured instrument response of band 27 in early mission but became negative values and much larger in magnitude in late mission for most of detectors. The approach is applied to both Blackbody calibration and MODIS L1B product. With the approach applied, the calibration coefficients derived from BB calibration become much smoother and detector differences of the coefficients are smaller. The crosstalk impact on band 27 brightness temperature can be as large as several degrees for a couple of detectors. The crosstalk effect made Baja peninsula clearly observed and introduced strong striping in recently Terra band 27's images. With the developed crosstalk algorithms applied, the dark shade of Baja peninsula induced by the crosstalk contributions mainly from band 29, a land band, is significantly removed in Terra band 27's images and the striping is substantially reduced. Examples selected in early, middle, and late missions are shown to demonstrate that the approach works remarkably well. The approach developed in this report for removal of the electronic crosstalk impact can be applied to other MODIS bands as well as to other instruments.

\section{ACKNOWLEDGEMENTS}

We would like to thank Chris Moeller, Aisheng Wu, and Bill Barnes for their helpful comments and suggestions.

\section{REFERENCES}

1. W.L. Barnes and V.V. Salomonson, "MODIS: A global image spectroradiometer for the Earth Observing System,” Critical Reviews of Optical Science and Technology, CR47, 285-307, 1993.

2. W.L. Barnes, V.V. Salomonson, B. Guenther, and X. Xiong, "Development, Characterization, and Performance of the EOS MODIS Sensors," Proceedings of SPIE - Earth Observing Systems VIII, 5151, 337-345, 2003.

3. V.V. Salomonson, W.L. Barnes, X. Xiong, S. Kempler, and E. Masuoka, "An Overview of the Earth Observing System MODIS Instrument and Associated Data Systems Performance,” Proceedings of IGARSS, 2002.

4. X. Xiong, J. Sun, W. Barnes, V. Salomonson, J. Esposito, H. Erives, and B. Guether, "Multiyear on-orbit calibration and performance of Terra MODIS reflective solar bands," IEEE Trans. Geosci. Remote Sensing, vol. 45, no. 4, 879-889, 2007.

5. Xiong, X., J. Sun, X. Xie, W. Barnes, and V. Salomonson, On-Orbit Calibration and Performance of Aqua MODIS Reflective Solar Bands, IEEE Trans. Geosci. Remote Sens., 48(1), 535-546, 2010, doi:10.1109/TGRS.2009.2024307.

6. X. Xiong, K. Chiang, A. Wu, W. Barnes, B. Guenther, and V. Salomonson, "Multiyear on-orbit calibration and performance of Terra MODIS thermal emissive bands,” IEEE Trans. Geosci. Remote Sensing, vol. 46, no. 6, 1790-1803, 2008.

7. X. Xiong, B. Wenny, A. Wu, W.L. Barnes, and V. Salomonson, “Aqua MODIS Thermal Emissive Bands Onorbit Calibration, Characterization, and Performance,” IEEE Trans. Geosci. Remote Sensing, 47(3), 803-814, 2009. 
8. G. Galina, S. Platnick, M. D. King, P. A. Hubanks, M. J. Pavolonis, A. K. Heidinger, P. Yang, and B. A. Baum, “Multilayer Cloud Detection with the MODIS Near-Infrared Water Vapor Absorption Band”, J. Appl. Meteor. Climatol., 49, 2315-2333, 2010.

9. X. Xiong, J. Sun; K. Chiang, S. Xiong; W. Barnes, “MODIS on-orbit characterization using the Moon”, Proceedings of SPIE - Sensors, Systems, and Next-Generation Satellites VI, 4881, 299-307, 2002.

10. J. Sun,, X. Xiong, N. Che, and A. Angal, "Terra MODIS band 2 electronic crosstalk: cause, impact, and mitigation,” Proceedings of SPIE - Sensors, Systems, and Next-Generation Satellites XIV, Vol. 7826, 78261Y, 2010. [doi: 10.1117/12.864738]

11. J. Sun, X. Xiong, W. Barnes, and B. Guether, "MODIS reflective solar bands on-orbit lunar calibration,” IEEE Trans. Geosci. Remote Sensing, vol. 45, no. 7, 2383-2393, 2007.

\section{Figure Caption:}

Figure 1, MODIS LWIR focal plane.

Figure 2, Lunar Image observed by Terra MODIS band 27 detector 1 on July 22, 2008. The instrument's response is limited to be less than 200 .

Figure 3, summations of Terra band 27 responses over scans for lunar observation on July 22, 2008 with data limited to be less than 4000 .

Figure 4, Crosstalk coefficient for the crosstalk of Terra band 27 from band 28.

Figure 5, Crosstalk coefficients for the crosstalk of Terra band 27 form band 29.

Figure 6, Crosstalk coefficient for the crosstalk of Terra band 27 from band 30.

Figure 7, Summation over scans for Terra band 27 lunar view responses after crosstalk effect corrected. Same lunar observation as is shown in Figure 3.

Figure 8, Terra band 27 calibration coefficients, b1, trending for entire mission without the crosstalk correction. The dotted vertical lines correspond to the instruments events such as nadir door open, electronic configuration switch, safe mode, back in science mode, etc.

Figure 9, Terra band 27 calibration coefficients, b1, trending for entire mission with the crosstalk correction.

Figure 10, Terra band 27 calibration coefficients, b1, on scan basis trending in granule 2010174:1840 without crosstalk correction.

Figure 11, Terra band 27 calibration coefficients, b1, on scan basis trending in granule 2010174:1840 with crosstalk correction.

Figure 12, Terra MODIS band 27 EV image on June 23, 2010 at Baja, California without crosstalk correction.

Figure 13, Terra MODIS band 27 EV image on June 23, 2010 at Baja, California with crosstalk correction.

Figure 14, Brightness temperature along scan direction observed by detector 1 with "Detector +10 x Scan” being 720 in Figures 12 and 13.

Figure 15, Brightness temperature along track direction with frame number 890 in Figures 12 and 13.

Figure 16, Terra MODIS band 27 EV image on November 19, 2006 at Baja, California without crosstalk correction.

Figure 17, Terra MODIS band 27 EV image on November 19, 2006 at Baja, California with crosstalk correction. 
Figure 18, Brightness temperature along scan direction observed by detector 1 with “Detector +10 x Scan” being 2230 in Figures 16 and 17.

Figure 19, Brightness temperature along track direction with frame number 716 in Figures 12 and 13.

Figure 20, Terra MODIS band 27 EV image on November 19, 2006 at Baja, California without crosstalk correction.

Figure 21, Terra MODIS band 27 EV image on November 19, 2006 at Baja, California with crosstalk correction.

Figure 22, Brightness temperature along scan direction observed by detector 1 with “Detector + 10 x Scan” being 1750 in Figures 20 and 21.

Figure 23, Brightness temperature along track direction with frame number 906 in Figures 20 and 21.

\section{LWIR}

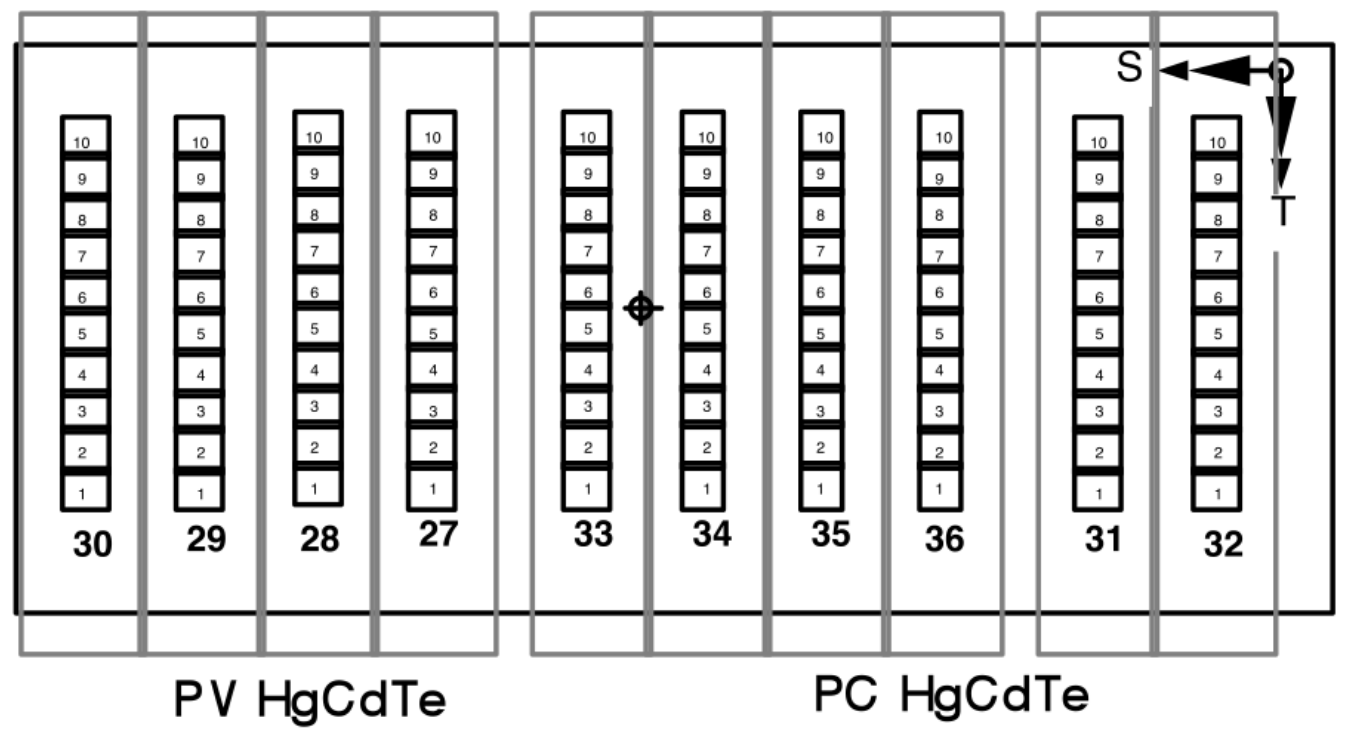

Figure 1, MODIS LWIR focal plane. 


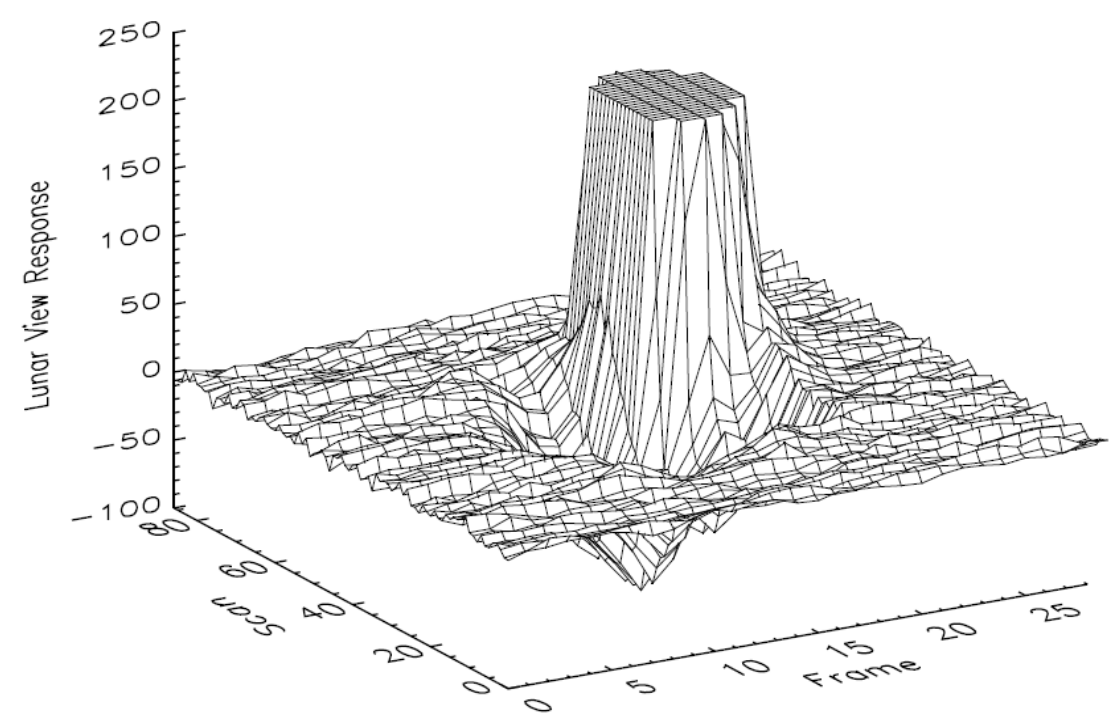

Figure 2, Lunar Image observed by Terra MODIS band 27 detector 1 on July 22, 2008. The instrument's response is limited to be less than 200 .

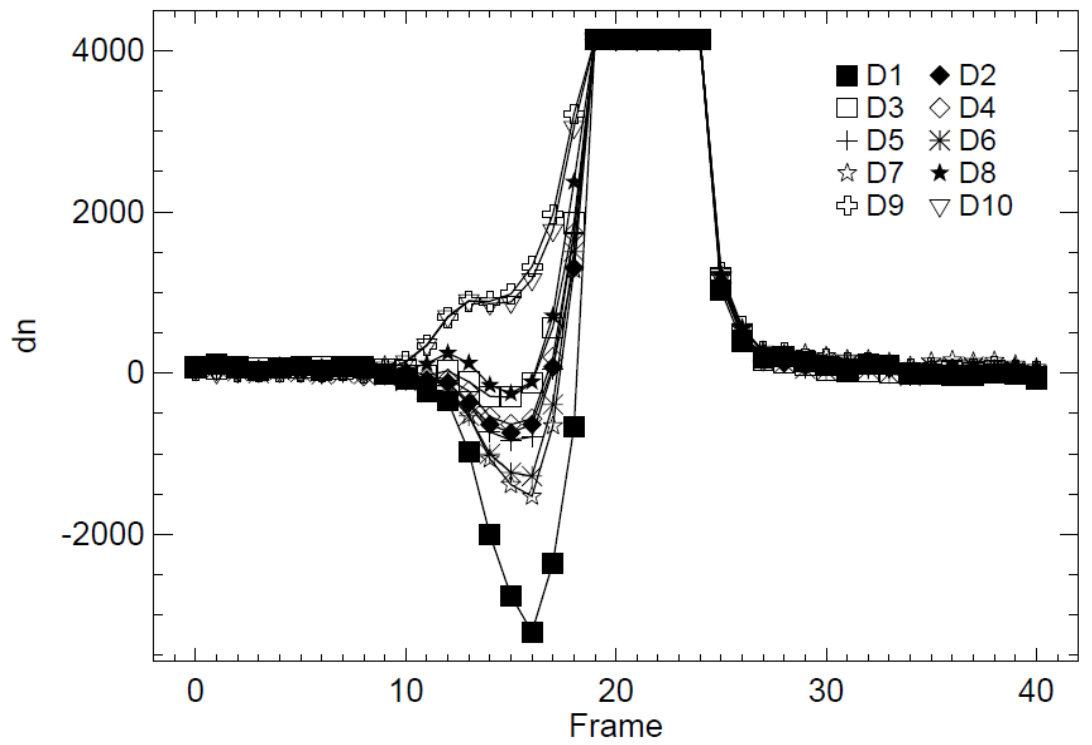

Figure 3, summations of Terra band 27 responses over scans for lunar observation on July 22, 2008 with data limited to be less than 4000 . 


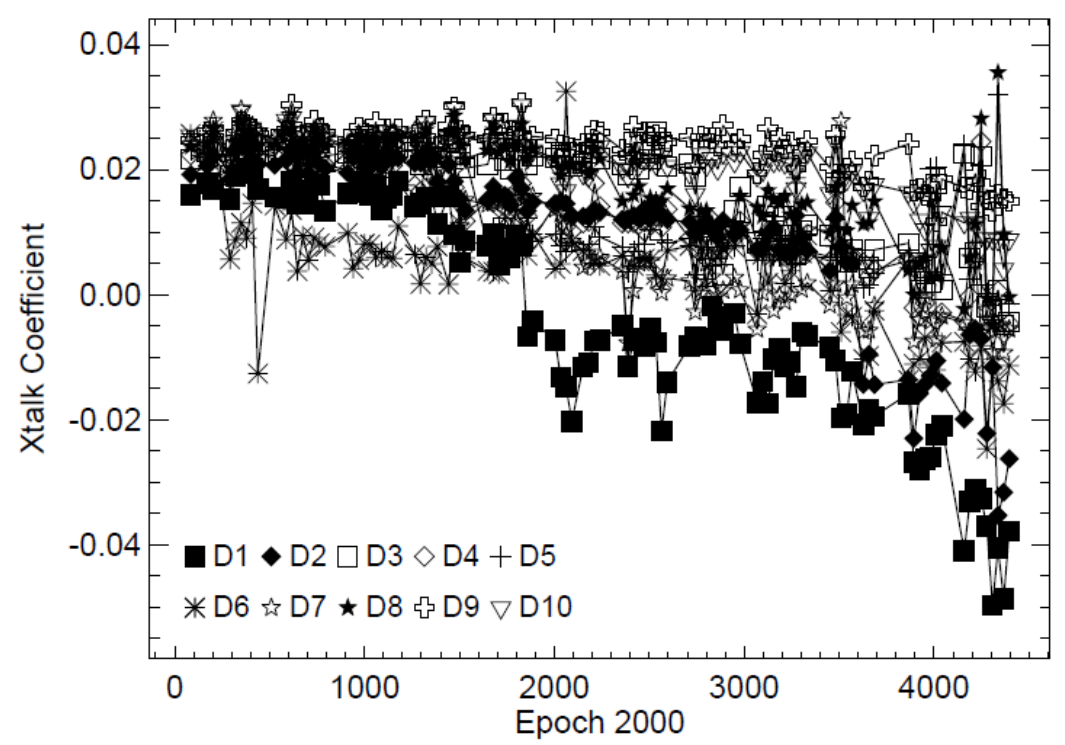

Figure 4, Crosstalk coefficient for the crosstalk of Terra band 27 from band 28.

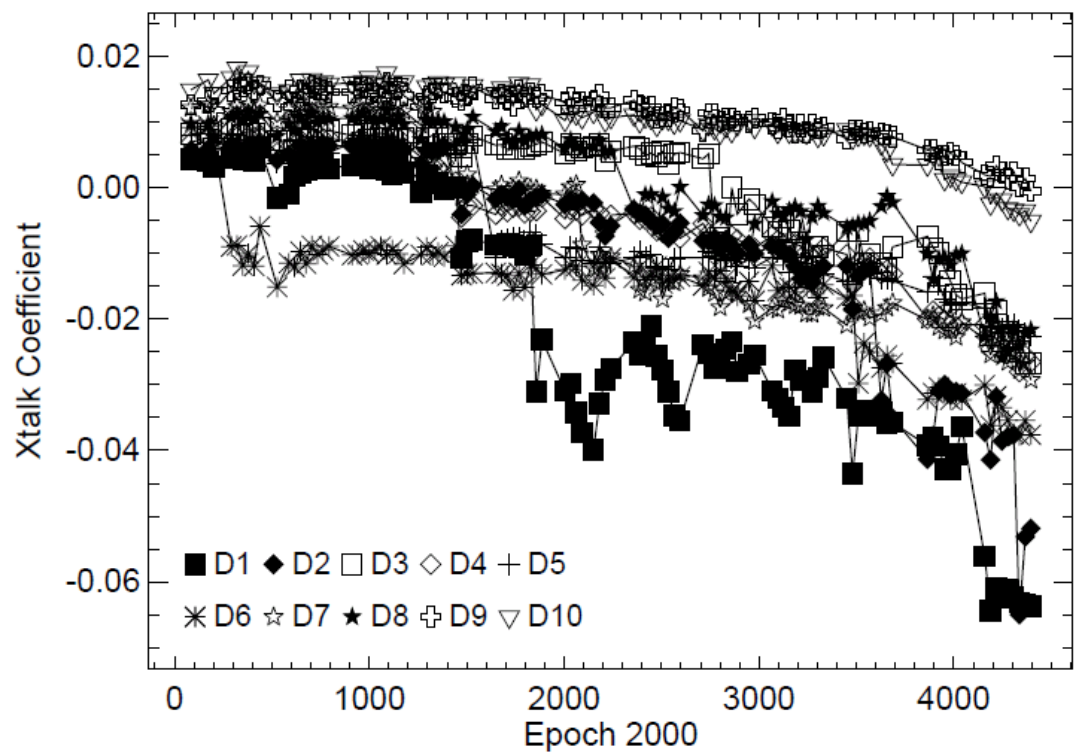

Figure 5, Crosstalk coefficients for the crosstalk of Terra band 27 from band 29. 


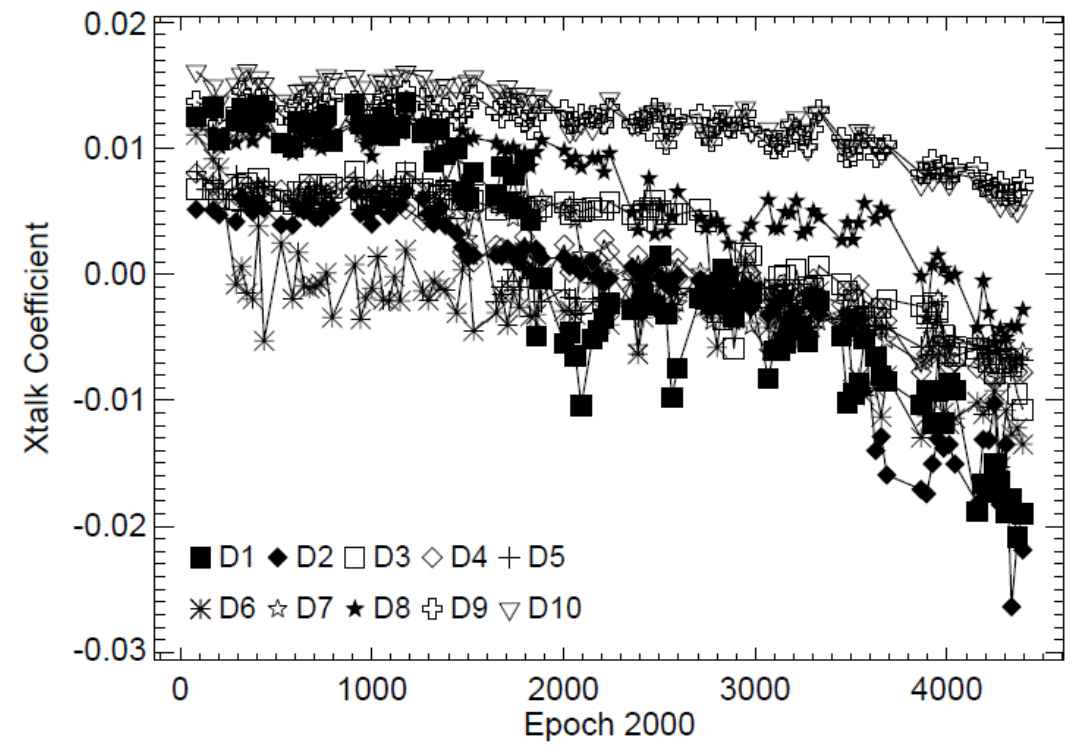

Figure 6, Crosstalk coefficient for the crosstalk of Terra band 27 from band 30 .

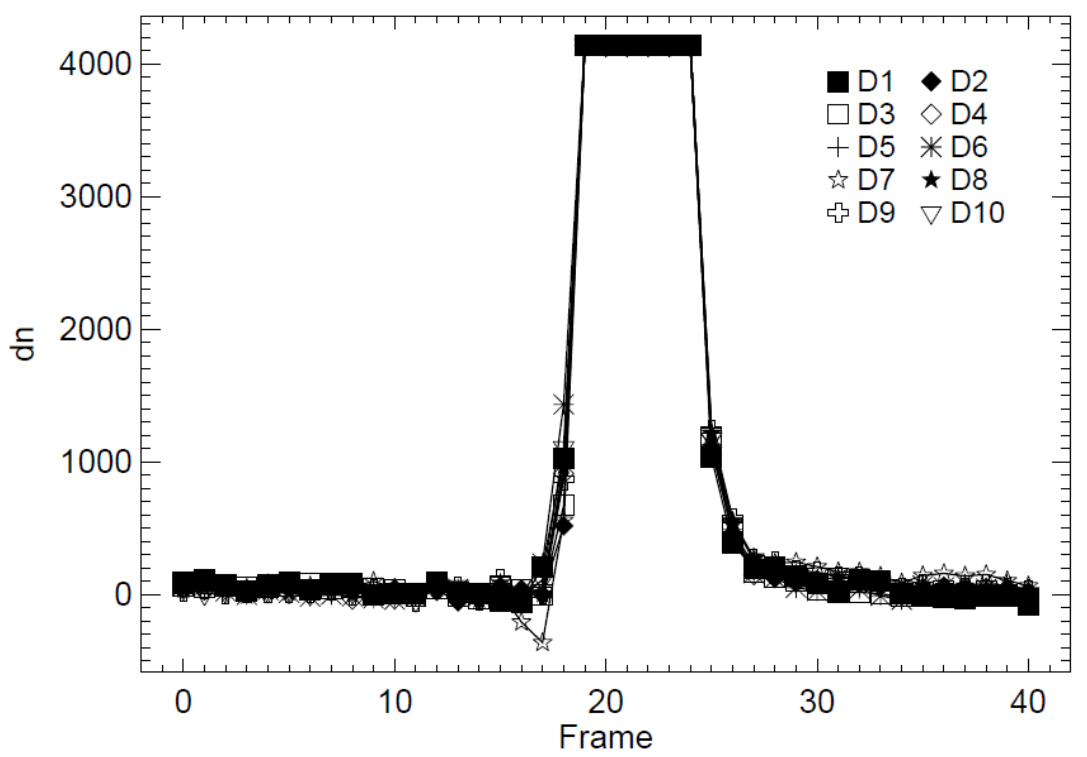

Figure 7, Summation over scans for Terra band 27 lunar view responses after crosstalk effect corrected. Same lunar observation as is shown in Figure 3. 


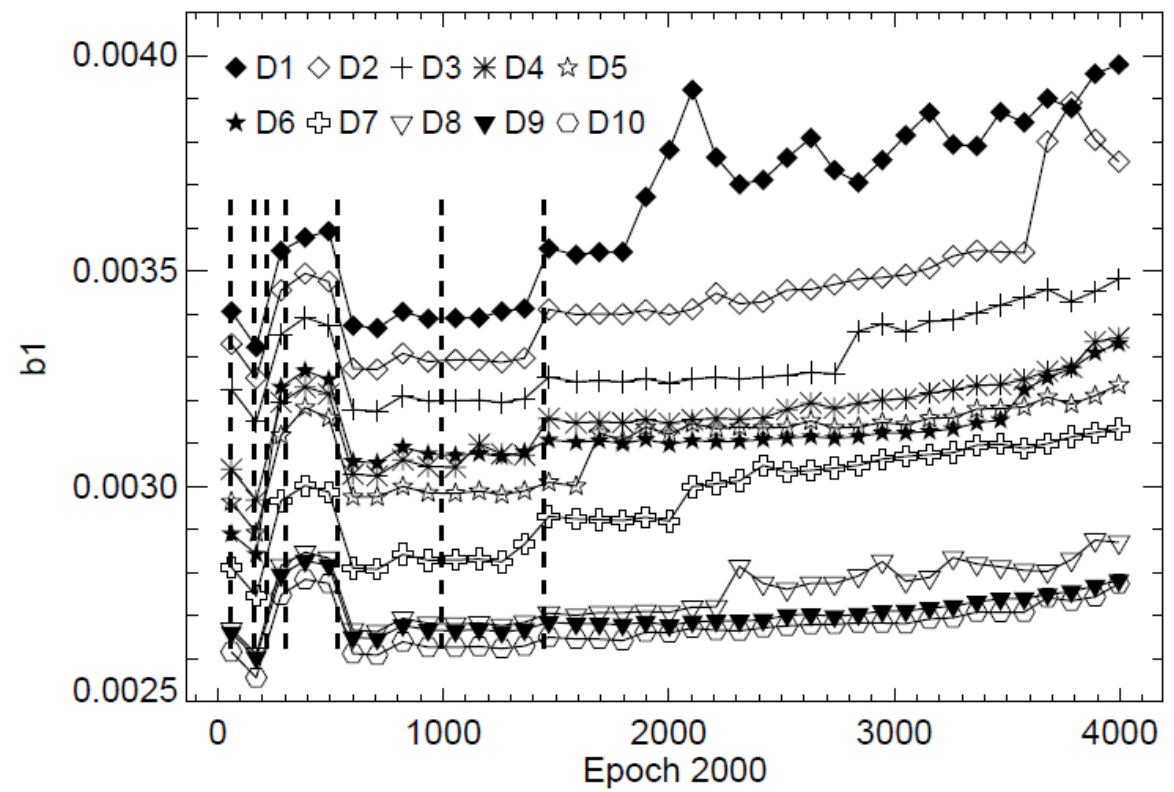

Figure 8, Terra band 27 calibration coefficients, b1, trending for entire mission without the crosstalk correction. The dotted vertical lines correspond to the instruments events such as nadir door open, electronic configuration switch, safe mode, back in science mode, etc.

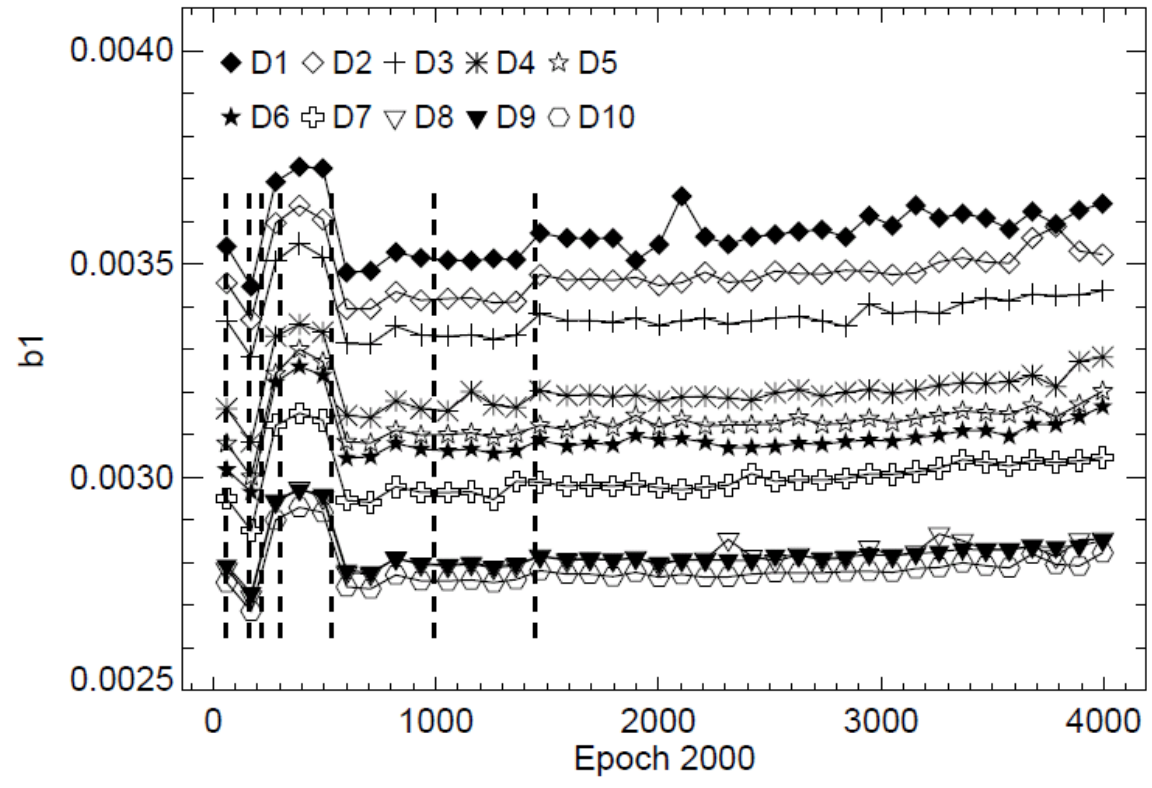

Figure 9, Terra band 27 calibration coefficients, b1, trending for entire mission with the crosstalk correction. 


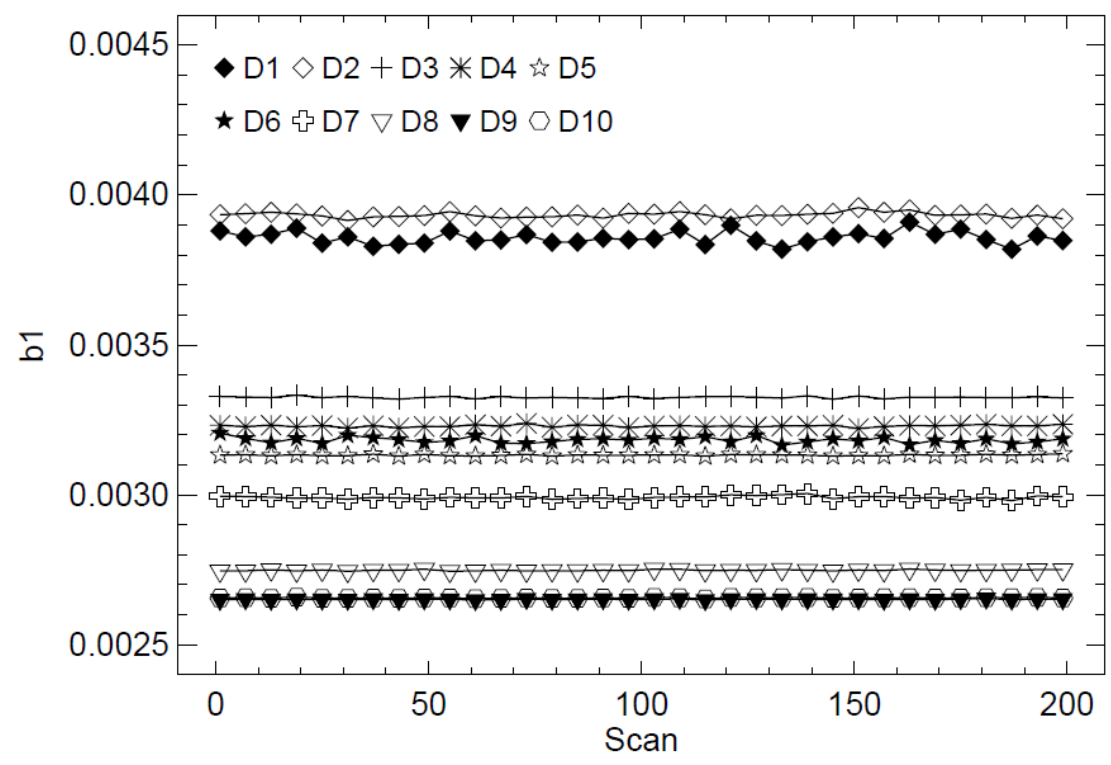

Figure 10, Terra band 27 calibration coefficients, b1, on scan basis trending in granule 2010174:1840 without crosstalk correction.

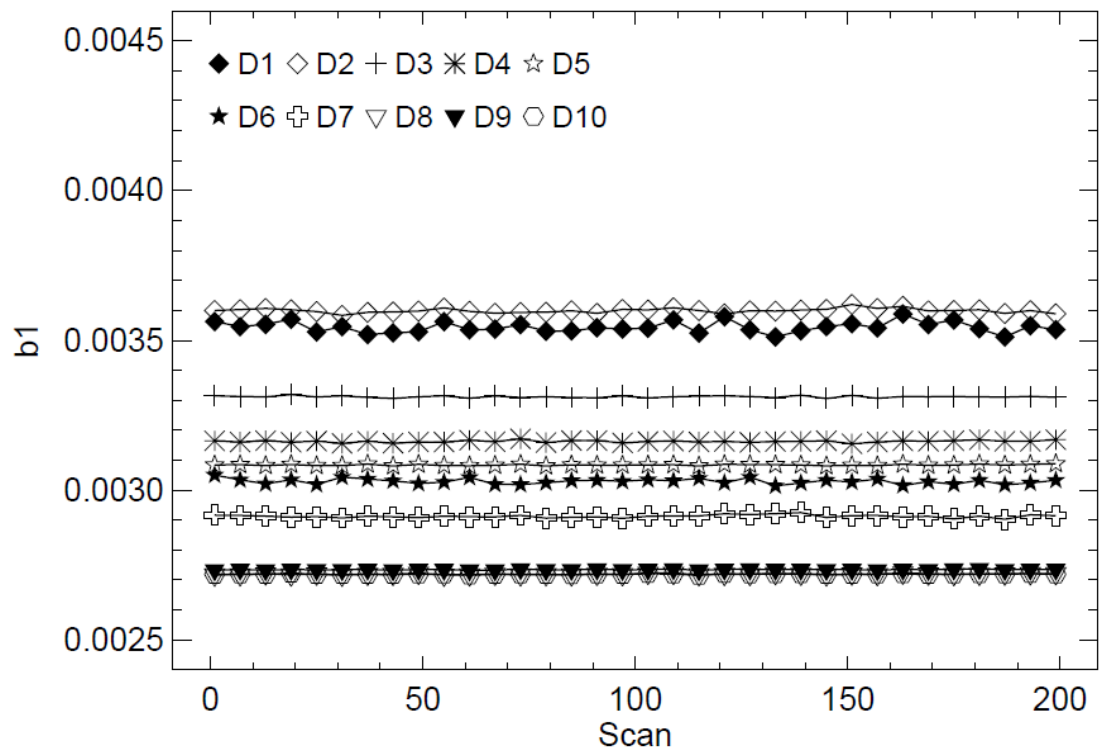

Figure 11, Terra band 27 calibration coefficients, b1, on scan basis trending in granule 2010174:1840 with crosstalk correction. 


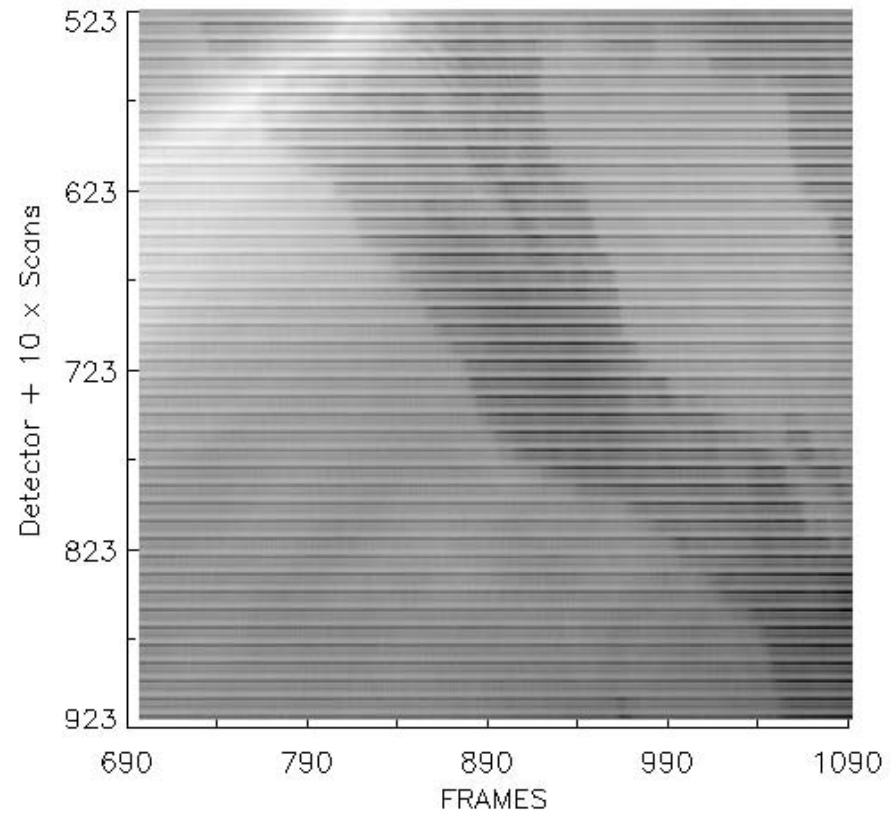

Figure 12, Terra MODIS band 27 EV image on June 23, 2010 at Baja, California without crosstalk correction.

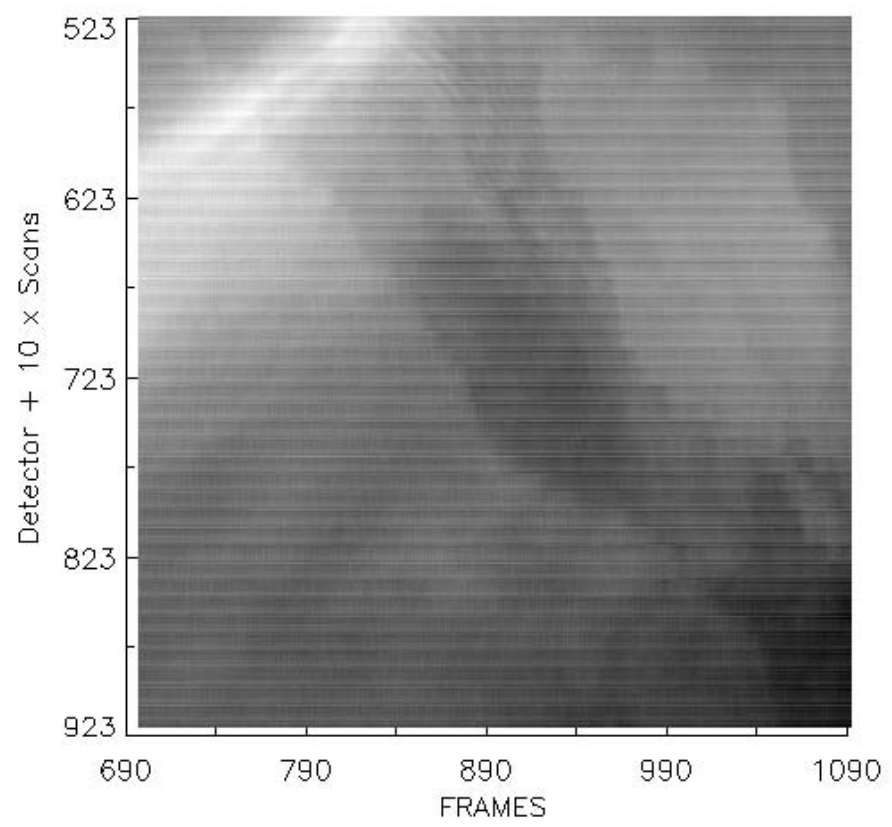

Figure 13, Terra MODIS band 27 EV image on June 23, 2010 at Baja, California with crosstalk correction 


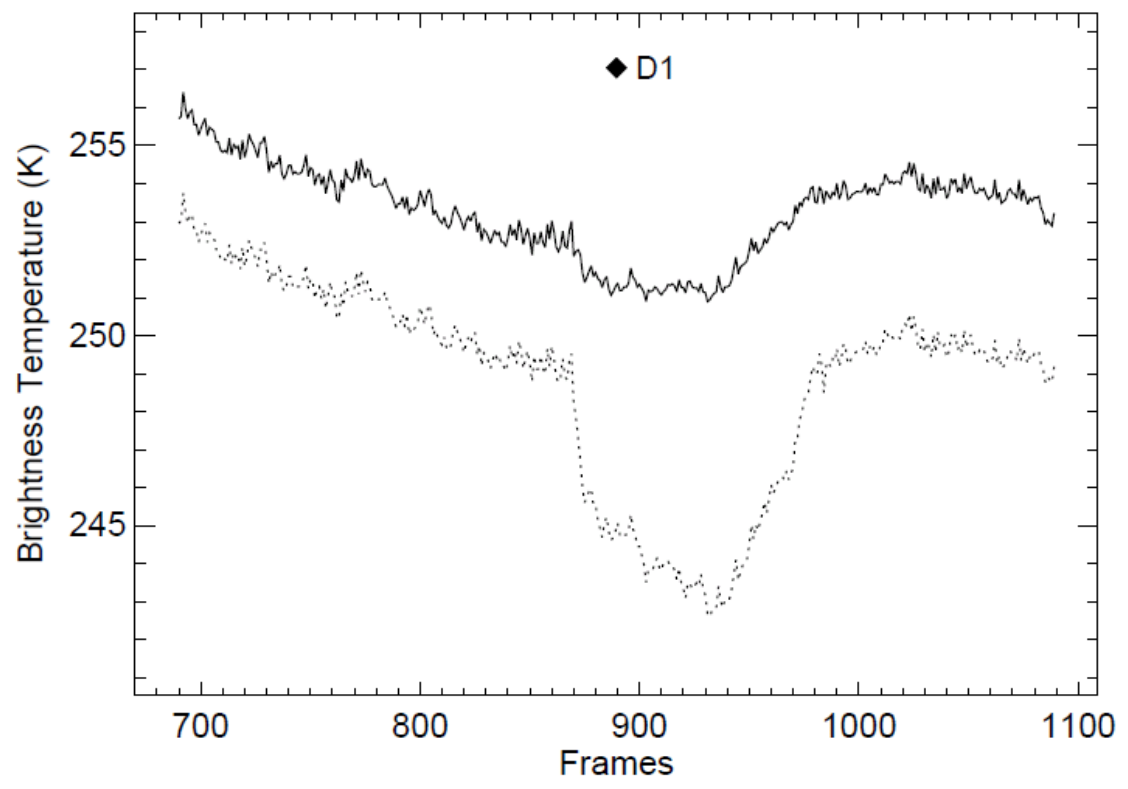

Figure 14, Brightness temperature along scan direction observed by detector 1 with "Detector +10 x Scan” being 720 in Figures 12 and 13.

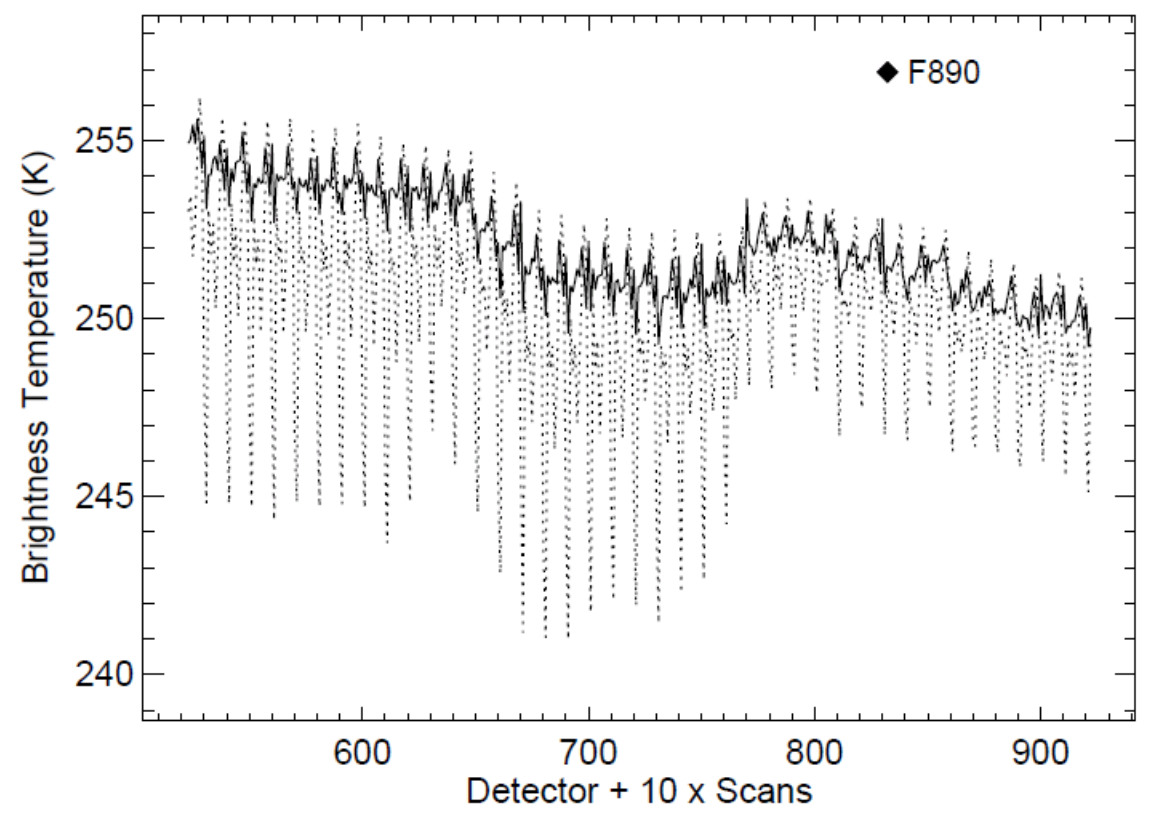

Figure 15, Brightness temperature along track direction with frame number 890 in Figures 12 and 13. 


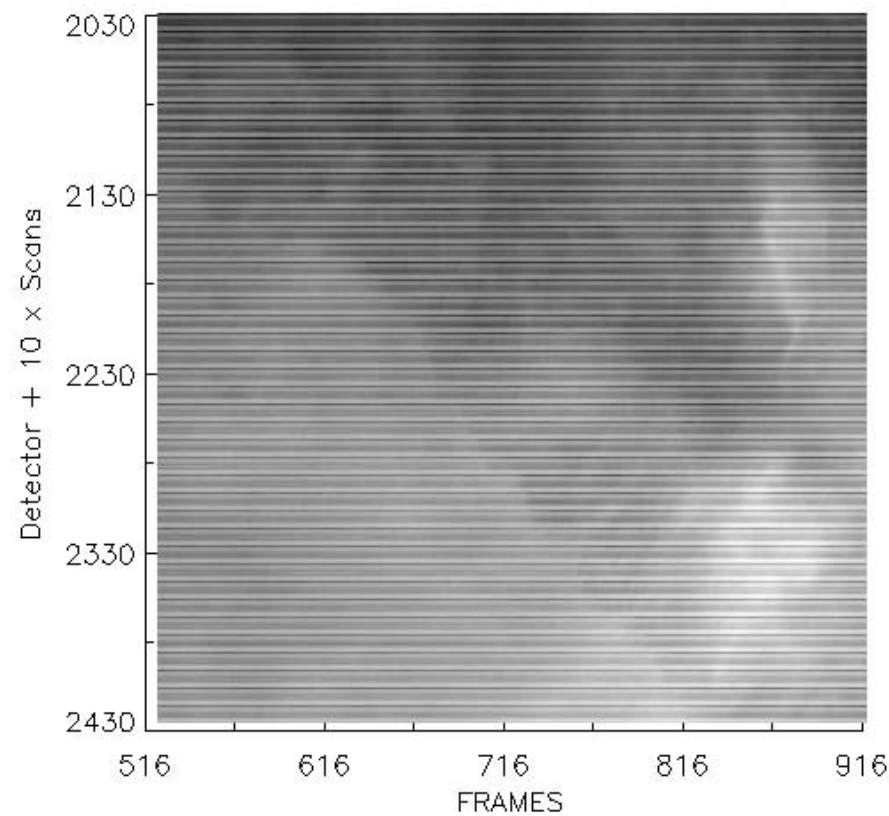

Figure 16, Terra MODIS band 27 EV image on November 19, 2006 at Baja, California without crosstalk correction.

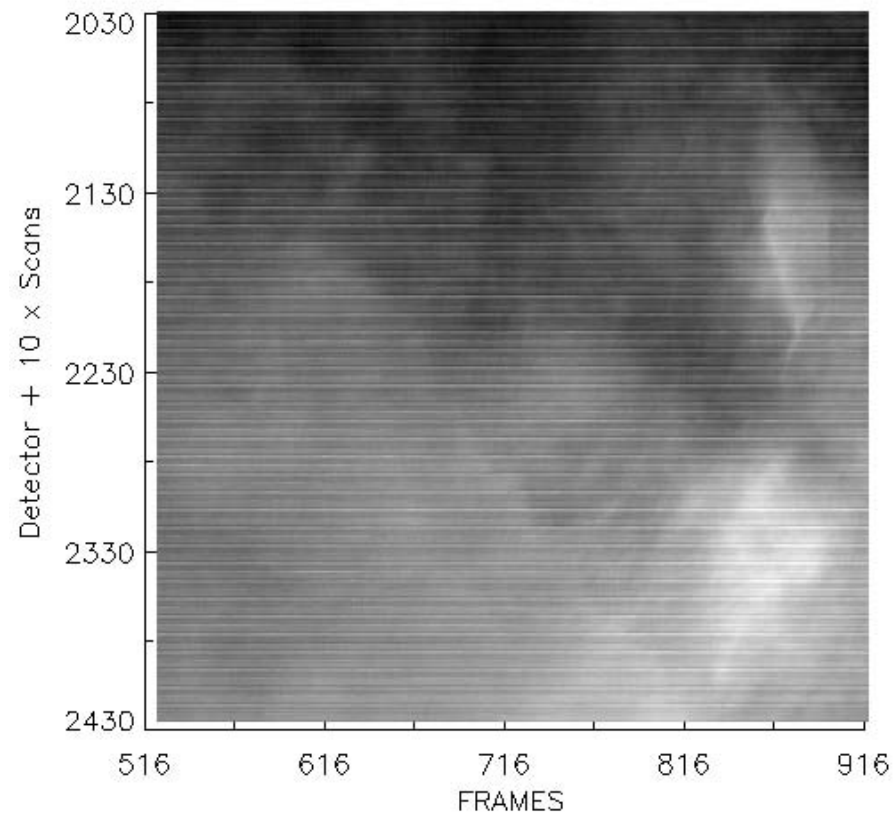

Figure 17, Terra MODIS band 27 EV image on November 19, 2006 at Baja, California with crosstalk correction. 


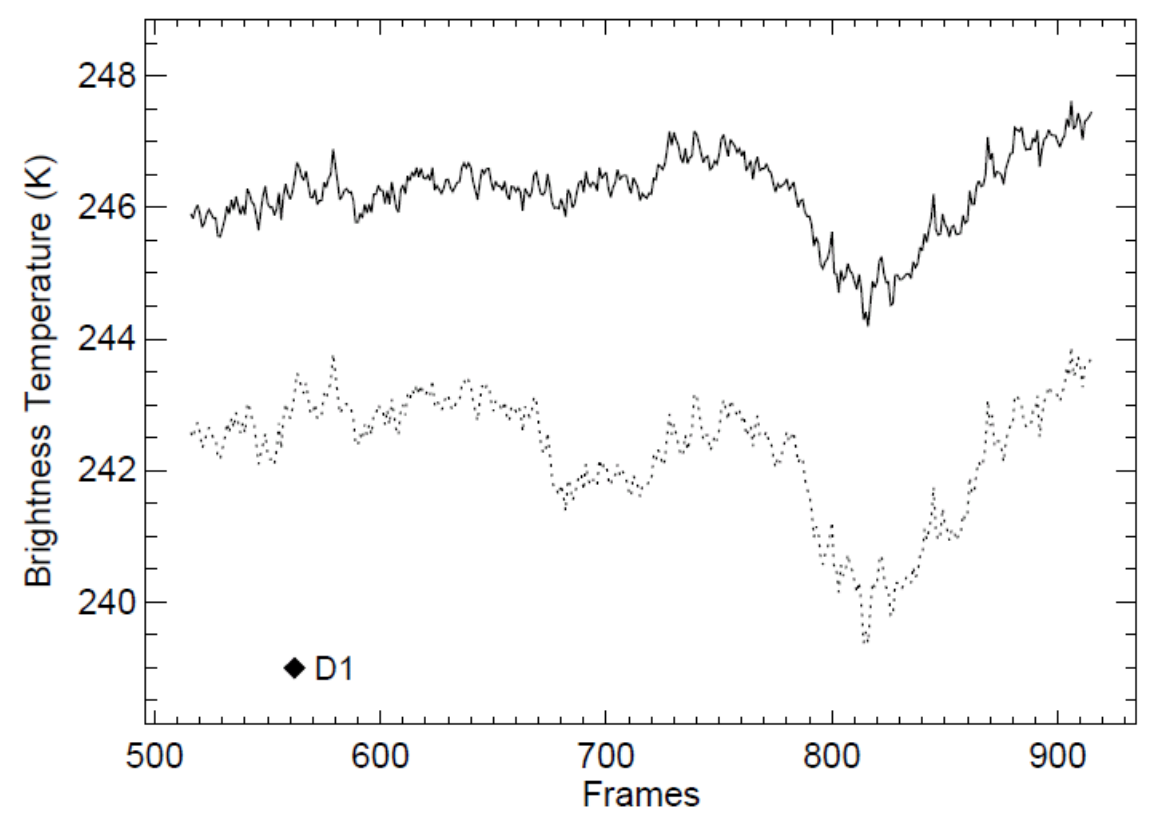

Figure 18, Brightness temperature along scan direction observed by detector 1 with "Detector +10 x Scan” being 2230 in Figures 16 and 17.

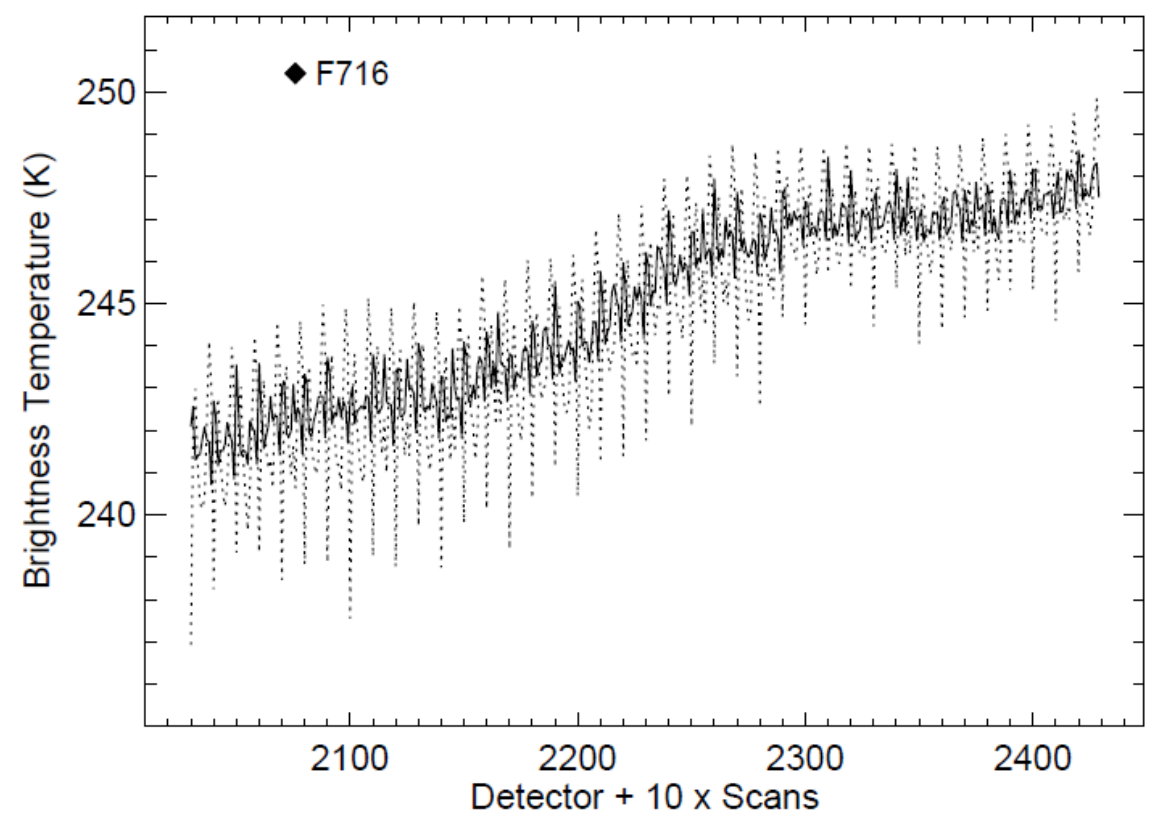

Figure 19, Brightness temperature along track direction with frame number 716 in Figures 12 and 13. 


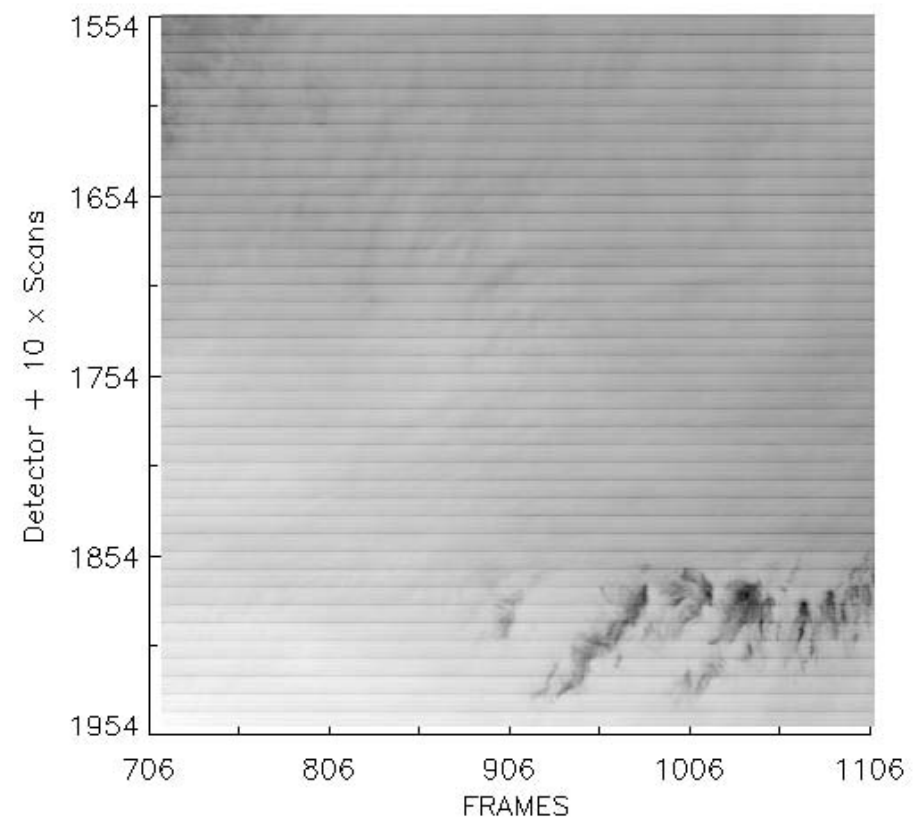

Figure 20, Terra MODIS band 27 EV image on December 23, 2001 at Baja, California without crosstalk correction.

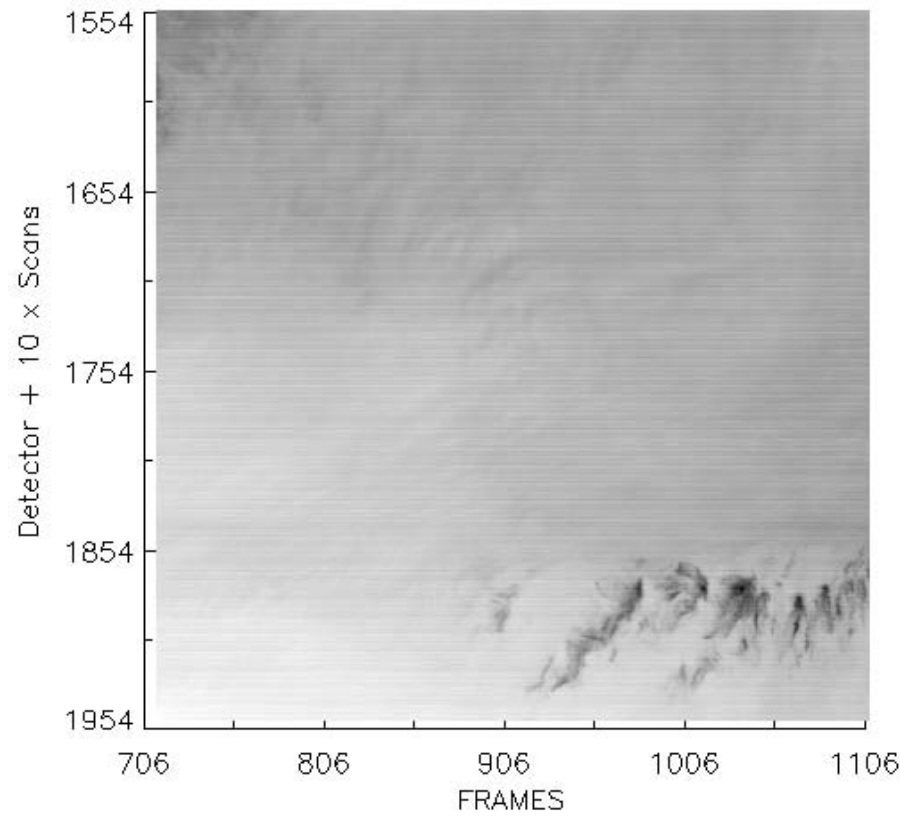

Figure 21, Terra MODIS band 27 EV image on December 20, 2000 at Baja, California with crosstalk correction. 


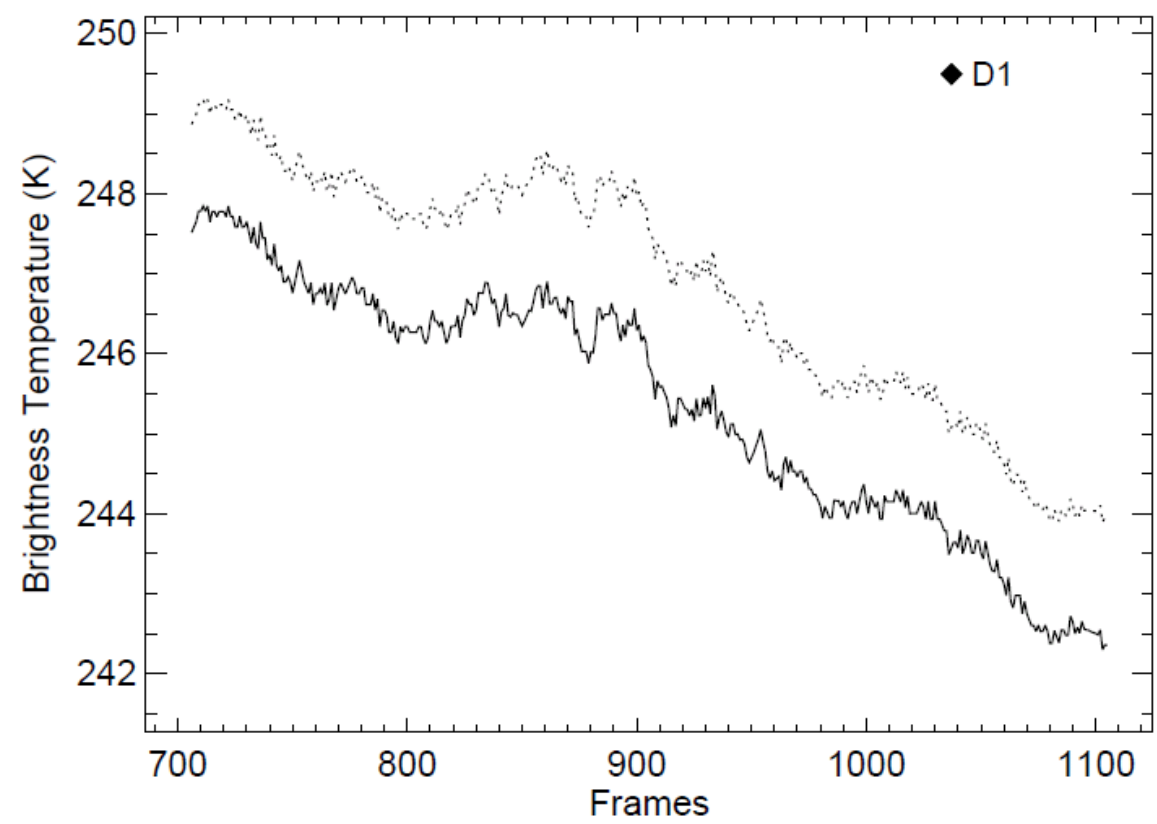

Figure 22, Brightness temperature along scan direction observed by detector 1 with "Detector +10 x Scan" being 1750 in Figures 20 and 21.

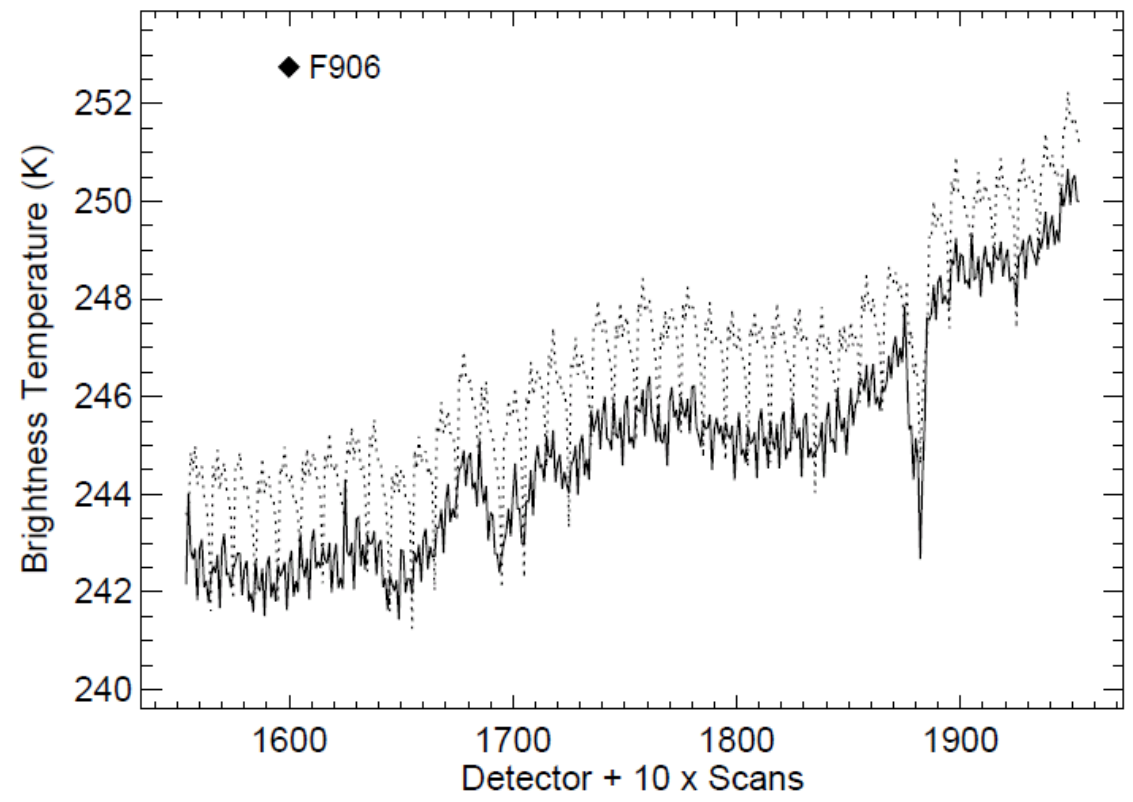

Figure 23, Brightness temperature along track direction with frame number 906 in Figures 20 and 21. 
